\title{
Intracranial Abscesses: An Institutional Study
}

\author{
Yogendra Singh*, Tarun Kumar Gupta, Gaurav Jaiswal, Krishna Lodha
}

Neurosurgery Department, R.N.T Medical College Udaipur, Rajasthan, India

Email: ${ }^{\star}$ Yogiforu0699@gmail.com

How to cite this paper: Singh, Y., Gupta, T.K., Jaiswal, G. and Lodha, K. (2020) Intracranial Abscesses: An Institutional Study. Open Journal of Modern Neurosurgery, 10, 297-306.

https://doi.org/10.4236/ojmn.2020.102032

Received: March 20, 2020

Accepted: April 21, 2020

Published: April 24, 2020

Copyright (อ 2020 by author(s) and Scientific Research Publishing Inc. This work is licensed under the Creative Commons Attribution International License (CC BY 4.0).

http://creativecommons.org/licenses/by/4.0/

(c) (i) Open Access

\begin{abstract}
Background: Brain abscess is defined as a focal intracranial infection that is initiated as an area of cerebritis and evolves into a collection of pus surrounded by a vascularized capsule. These are complications of head trauma, neurosurgical operations, meningitis, and otogenic, mastoid, and paranasal air sinus infections. Management involves both medical and surgical treatment. Surgical management includes either aspiration or excision of lesions larger than $2.5 \mathrm{~cm}$ in diameter, depending on brain location. However, literature on surgical treatment is replete with several procedures which, on their own, may not determine outcome. Aim: Aim is to study the epidemiology, management and outcome of various treatment modalities of brain abscess in our institute. Material and Methods: We conducted a retrospective study of demographic data as well as indications, treatment modalities, and outcomes of various surgical procedures for evacuation of intracranial abscesses in patients admitted to our Neuro-intensive care unit at R.N.T. Medical College, Udaipur, Rajasthan, India from January 2013 to June 2019. Results: We carried out 53 procedures in 43 (30 male and 13 female) patients with various intracranial abscesses. Most abscesses [16, i.e. $37 \%]$ occurred in the second decade and second most common in the first decade [15, i.e. 34\%]. In infants 4 (9\%) cases of intracranial abscesses were present. None of the infants had features of congenital heart disease. The predisposing factors were mostly otolaryngologic (15) or posttraumatic (8). Most commonly abscesses were located in frontal 13 (30\%) followed by cerebellar 9 (21\%). Burr hole evacuation was done in $74 \%$ of cases. 3 patients ( $7 \%$ of cases) died. Prognosis appears to worsen with ventriculitis, multiple abscesses especially in infants, and immunosuppression. Conclusion: In conclusion, brain abscess still continues to be a formidable challenge, with prognosis that dramatically improved over the last decades due to advances in brain imaging, neurosurgical techniques and better use of old and more recent antibacterial agents. Mortality is improved compared with historical series; however, long-term morbidity is significant particularly in the infant population. Further researches
\end{abstract}


must be conducted to clarify specific aspects, such as anticonvulsant prophylaxis/therapy, and also for the improvement of microbiological diagnosis.

\section{Keywords}

Brain Abscess, Aspiration, Cerebritis

\section{Introduction}

Brain abscess is defined as a focal intracranial infection that is initiated as an area of cerebritis and evolves into a collection of pus surrounded by a vascularized capsule. Magnetic resonance imaging (MRI) is the diagnostic neuroimaging procedure of choice in patients with brain abscess [1]. It is more sensitive than computed tomography (CT) and offers significant advantages in the early detection of cerebritis, more conspicuous demonstration of spread of inflammation into the ventricles and subarachnoid space, and earlier detection of satellite lesions [2]. This paper is a study of pattern and outcomes of treatments of intracranial abscesses in our institute as well as review of available literature on the subject.

\section{Materials and Methods}

We conducted a retrospective study of demographic data as well as indications, treatment modalities, and outcomes of various surgical procedures for evacuation of intracranial abscesses in patients admitted to our Neuro-intensive care unit at R.N.T. Medical College, Udaipur, Rajasthan, India from January 2013 to June 2019. Ethical clearance for this study has been taken. Demographic, clinical, and radiological data, treatment modalities, and outcomes were obtained from case file and radiological and operating room records. All patients had detailed neurological and systemic examination to find sources of infection. CT/MRI scan was carried out on all patients on the finding of clinical features referable to the nervous system or features of raised intracranial pressure. A hypodense mass with an encircling ring of contrast enhancement - usually associated with perilesional edema and mass effect - on brain CT/MRI confirms the diagnosis of abscess. The patients were thereafter prepared for emergency evacuation.

\section{Laboratory Investigations}

Full blood count, erythrocyte sedimentation rate, serum electrolytes, urea and creatinine, blood sugar, Mantoux test, and retroviral screening were requested, if indicated, especially in recurrent abscesses and very ill patients. Otorhinolaryngologic assessment was carried out on patients with history of or ongoing sinus and ear infections. Chest radiographs were requested for all patients and children below 2 years, who were further examined with echocardiography. 43 patients underwent various surgical evacuation procedures, viz. burr hole evacua- 
tion, limited craniectomy procedures with abscess evacuation in patients with associated osteomyelitis of the skull, bedside needle aspiration via previous burr hole or cranial defect and craniotomy with decortication. The patients were commenced on broad-spectrum antibiotics: Intravenous ceftriaxone and gentamycin and, in patients with otogenic infections, metronidazole was added for 6 8 weeks, followed by 6 - 8 weeks of oral antibiotic treatment depending on the culture reports.

\section{Results}

43 patients underwent 53 surgical procedures for evacuation of intracranial abscesses. The ages of patients ranged from 3 months to 75 years (mean: 17.5 years). There were 30 males and 13 females $(\mathrm{M}: \mathrm{F}=2.3: 1)$. Most intracranial abscesses occurred in the second decade of life, accounting for 16 , i.e. $37 \%$, followed by in the first decade of life i.e. 15 (34\%) (Table 1). Most common location was frontal 13 (30\%), followed by cerebellar 9 (21\%). Most common procedure done was burr hole evacuation.

\subsection{Predisposing Factors}

The source of the abscess was unknown in 13 patients. The others were: Post-meningitic (10), otogenic (2), mastoiditis (2), sinusitis (6), posttraumatic (6) and tuberculosis (4) (Table 2).

\subsection{Location and Type}

13 (30\%) intracranial abscess were frontal in location. The other locations were: Parietal (6), temporal (4), fronto-parietal (1), temporo-parietal (3), parieto-occipital (6), cerebellar (9), fronto-temporal (1) (Table 3).

Table 1. Age and sex distribution of intracranial abscesses.

\begin{tabular}{cccc}
\hline Age ( years) & Male & Female & Total \\
\hline $0-1$ & 3 & 1 & 4 \\
$2-5$ & 7 & 0 & 7 \\
$6-10$ & 3 & 1 & 4 \\
$11-20$ & 11 & 5 & 16 \\
$21-30$ & 3 & 1 & 4 \\
$31-40$ & 3 & 1 & 4 \\
$41-50$ & 0 & 2 & 2 \\
$51-60$ & 0 & 0 & 0 \\
$>60$ & 0 & 2 & 2 \\
Total & 30 & 13 & 43 \\
\hline
\end{tabular}


Table 2. Predisposing factors.

\begin{tabular}{cc}
\hline Source of infection & Total no \\
\hline unknown & 13 \\
Post-meningitic & 10 \\
otogenic & 2 \\
mastoiditis & 2 \\
sinusitis & 6 \\
posttraumatic & 6 \\
tuberculosis & 4 \\
total & 43 \\
\hline
\end{tabular}

Table 3. Location of abscesses.

\begin{tabular}{cc}
\hline Location & No (\%) \\
Frontal & $13(30)$ \\
Parietal & $6(15)$ \\
temporal & $4(9)$ \\
Fronto-parietal & $1(2)$ \\
Fronto-temporal & $1(2)$ \\
Temporo-parietal & $3(6)$ \\
Parieto-occipital & $6(15)$ \\
cerebellar & $9(21)$ \\
Total & 43 \\
\hline
\end{tabular}

\subsection{Hemispheric Distribution}

17 were located in the right hemisphere and 26 were located in left hemisphere (Table 4 and Figures 1-5).

\subsection{Surgical Treatment}

Burr hole evacuation was carried out in 32 patients - frontal (14), parietal (10), temporal (6), frontal and parietal (1) (Table 5). Aspiration of residual abscess was repeated in 10 patients. Limited ( $3-6 \mathrm{~cm}$ diameter) craniectomy procedures with abscess evacuation were carried out in patients with associated osteomyelitis of the skull with or without depressed skull fracture: suboccipital craniectomy evacuation, frontal craniectomy evacuation with elevation of depressed fracture [2], craniectomy was performed for all cerebellar abscess, 9 out of 9 (Table 6). Burr hole evacuation was sufficient for most of the abscess located in cerebral hemisphere except when associated with skull osteomyelitis or fracture of skull bone. 
Table 4. Hemispheric distribution.

\begin{tabular}{cl}
\hline Right & 17 \\
Left & 26 \\
Total & 43 \\
\hline
\end{tabular}

Table 5. Location of burr holes.

\begin{tabular}{cc}
\hline Burr hole evacuation & no \\
\hline Frontal & 14 \\
parietal & 10 \\
temporal & 6 \\
Frontal and parietal & 2 \\
total & 32 \\
\hline
\end{tabular}

Table 6. Limited craniectomy.

\begin{tabular}{cc}
\hline Limited craniectomy $(3-6 \mathrm{~cm})$ & no \\
\hline Sub-occipital & 9 \\
Frontal craniectomy & 2 \\
Total & 11 \\
\hline
\end{tabular}

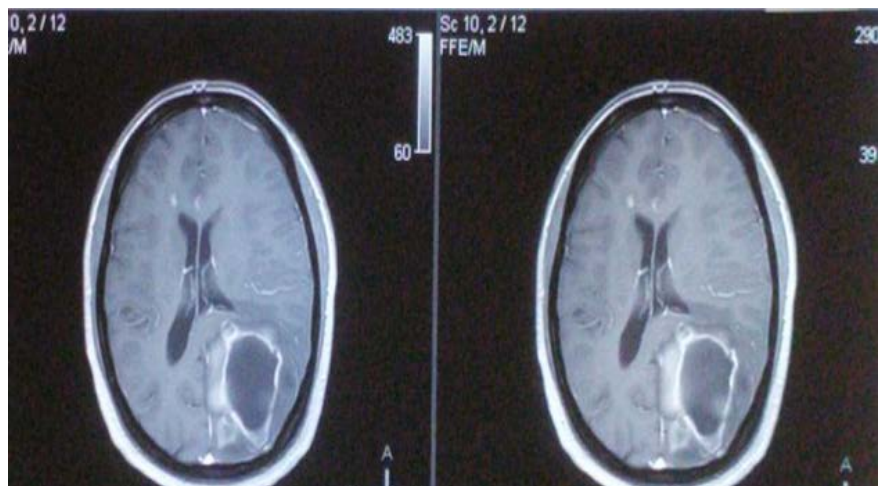

Figure 1. T1W post contrast MRI showing ring enhancing lesion in left parieto-occipital region.

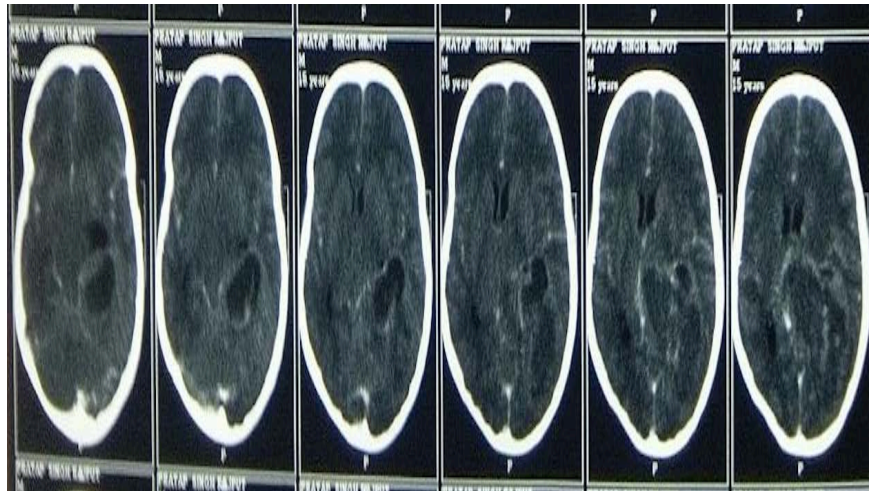

Figure 2. Post contrast CT scan showing ring enhancing lesion in left parietal region. 


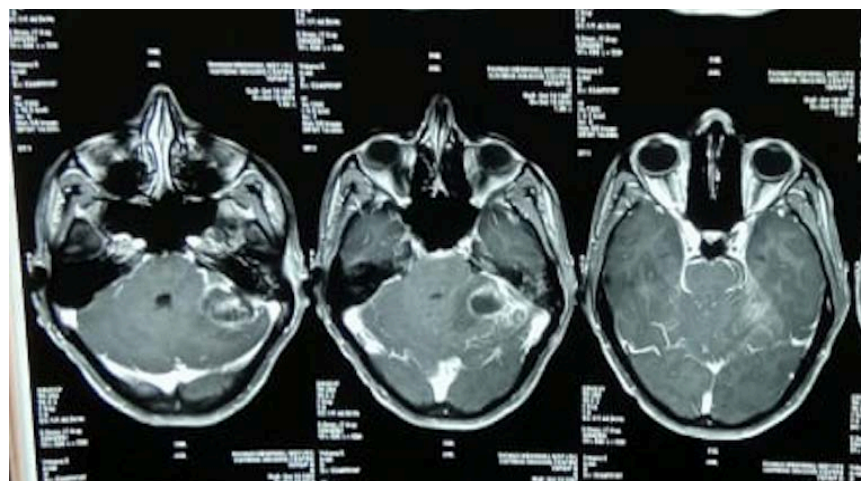

Figure 3. T1W post contrast MRI showing ring. Enhancing lesion in left cerebellar hemisphere.

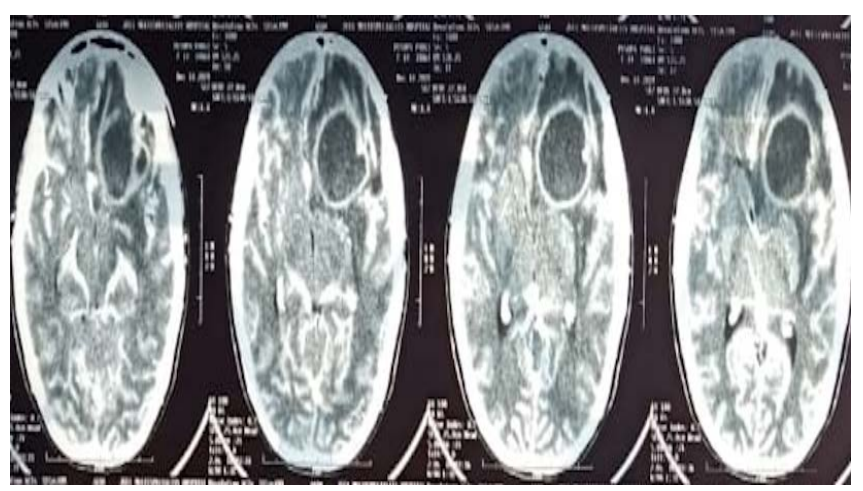

Figure 4. Post contrast CT scan showing ring enhancing lesion in left frontal lobe.

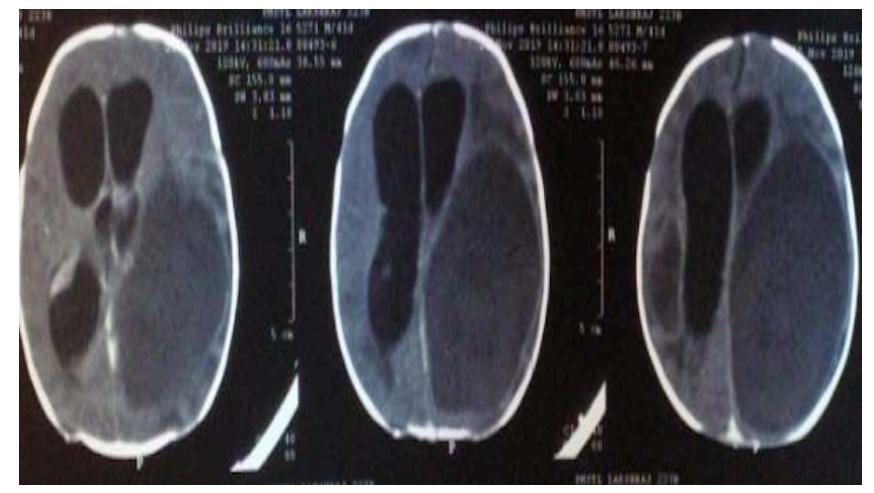

Figure 5. Non contrast CT scan in 5 month old male showing large abscess in left temporo parietal region.

\section{Discussion}

Brain abscess is defined as a focal intracranial infection that is initiated as an area of cerebritis and evolves into a collection of pus surrounded by a vascularized capsule. The most common predisposing conditions in children were sinusitis, meningitis, and traumatic brain injury [3]. Organisms can reach the central nervous system (CNS) through spread from a contiguous source of infection ( $25 \%$ to $50 \%$ of cases), hematogenous dissemination ( $20 \%$ to $35 \%$ of cases), 
or trauma [4] [5]. It can occur at any age (average ranges from 40 to 45 years), with a male to female ratio of 2:1 in most studies [6]. Our study showed a marked male predominance in $<40$ years and female predominance $>40$ years, there are more patients in second decade. Sources of a contiguous focus of infection include the middle ear, mastoid cells, and paranasal sinuses. Brain abscess that results from otitis media usually localizes to the temporal lobe or cerebellum; in one review, $54 \%$ were in the temporal lobe, $44 \%$ in the cerebellum, and $2 \%$ in both locations [7]. In patients with brain abscess secondary to paranasal sinusitis, the frontal lobe is the predominant site. When the abscess is a complication of sphenoid sinusitis, the temporal lobe or sella turcica is usually involved. Dental infections, particularly of the molar teeth, can lead to brain abscess [8] [9]; these often occur in the frontal lobe, but temporal lobe extension has been reported [10].

\subsection{Clinical Features}

Headache is generally observed in $70 \%$ to $75 \%$ of patients; sudden worsening of the headache, accompanied by new onset of meningismus, may signify rupture of the abscess into the ventricular space [11]. The classic triad, fever, headache, and focal neurological deficits, is seen in less than $50 \%$ of patients with brain abscess. The specific neurological findings for brain abscess are also defined by location within the CNS [12].

\subsection{Diagnosis}

The evolution of a brain abscess includes four stages based on histological criteria: The acute inflammatory stages, early cerebritis (days 1 - 3) and late cerebritis (days 4 - 9), early encapsulation (days 10 - 13), and late encapsulation (day 14 and later). In lesions that were well encapsulated (14 days and older), five distinct histological zones were apparent: A well-formed necrotic center; a peripheral zone of inflammatory cells, macrophages, and fibroblasts; the dense collagenous capsule; a layer of neovascularity associated with continuing cerebritis; and reactive astrocytes, gliosis, and cerebral edema external to the capsule [13]. The CT appearance of well-encapsulated abscesses showed a typical ring-shaped contrast-enhancing lesion. Magnetic resonance imaging (MRI) is the diagnostic neuroimaging procedure of choice in patients with brain abscess, it is more sensitive than computed tomography (CT) and offers significant advantages in the early detection of cerebritis, more conspicuous demonstration of spread of inflammation into the ventricles and subarachnoid space, and earlier detection of satellite lesions. On T1-weighted images, the abscess capsule often appears as a discrete rim that is isointense to mildly hyperintense; administration of gadolinium-diethylenetriaminepentaacetic acid helps clearly differentiate the central abscess, surrounding enhancing rim, and cerebral edema. On T2-weighted images, the zone of edema that surrounds the abscess demonstrates marked high signal intensity in which the capsule appears as an ill-defined hypointense rim at 
the margin of the abscess.

\subsection{Management}

The initial approach to management of a patient with a suspected brain abscess is a multidisciplinary one that involves a neuroradiologist, neurosurgeon, and infectious disease specialist. After neuroimaging, if single or multiple ring-enhancing lesions are found, prudent management involves either aspiration or excision of lesions larger than $2.5 \mathrm{~cm}$ in diameter, depending on brain location. Although abscess size greater than $2.5 \mathrm{~cm}$ has been recommended as an indicator for neurosurgical intervention, data from comparative studies are lacking, and this size cannot be regarded as a definitive indication for aspiration. Drainage should be considered if an abscess is abutting the ventricular system, to prevent abscess rupture and resulting ventriculitis. After aspiration of abscess material and submission of specimens for special stains, histopathologic examination, and culture, empirical antimicrobial therapy should be initiated. Because antimicrobial therapy before aspiration may reduce the yield of bacterial cultures, it is reasonable to postpone initiation of antimicrobial therapy until after neurosurgery has been performed. Delaying antimicrobial therapy should be considered only in clinically stable patients and, therefore, every effort should be made to perform surgery in an expedited manner. The combination of metronidazole plus a third-generation cephalosporin is commonly used, in patients in whom $S$. aureus is also considered a probable pathogen, vancomycin is added pending identification of the organism and in vitro susceptibility testing. Surgical therapy is often required for the optimal approach to patients with bacterial brain abscess [14] [15] [16] [17]. Procedures include bur-hole aspiration and complete excision after craniotomy, although no prospective trial comparing these two procedures has ever been performed. However, in one retrospective review of 47 studies from 1990 to 2008, patients who underwent aspiration had a mortality of $6.6 \%$, compared with $12.7 \%$ in those who underwent surgical excision, a second aspiration should be considered if the initial aspiration proves ineffective or partially effective. Mortality rates in patients with brain abscess in the pre-antibiotic era and into the 1970s were unacceptably high and ranged from $30 \%$ to $80 \%$. Since the 1970 s, case fatality rates have ranged from $0 \%$ to $24 \%$. This decrease has been attributed to the introduction ( 1970s) of neuroimaging, which allows early diagnosis and monitoring of response to therapy. Although the optimal approach to brain abscess most often requires a combined medical and surgical approach, certain groups of patients may be treated with medical therapy alone [18] [19]. Such groups include those with medical conditions that increase the risk associated with surgery, with multiple abscesses, with abscesses in a deep or dominant location, with coexisting meningitis and with abscess size less than $2.5 \mathrm{~cm}$ to $3 \mathrm{~cm}$. Complete excision by craniotomy is now infrequently performed because of the success of aspiration and closed-drainage techniques, although it may be required for patients with multiloculated ab- 
scesses in whom aspiration techniques have failed, for abscesses containing gas, or for abscesses that fail to resolve. Excision is usually required for posttraumatic abscesses that contain foreign bodies or retained bone fragments to prevent recurrence, for abscesses that result from fistulous communications (e.g., secondary to trauma or congenital dermal sinuses), and for those localized to one lobe of the brain and contiguous with a primary focus . It has also been suggested that excision is the preferred method of surgical treatment of cerebellar abscesses in children [20] given that worse outcomes have been seen in those treated only by aspiration. However, bur-hole aspiration has also been suggested as a satisfactory method of drainage in patients with cerebellar abscesses [21].

\section{Conclusion}

In conclusion, brain abscess still continues to be a formidable challenge, with prognosis that dramatically improved over the last decades due to advances in brain imaging, neurosurgical techniques and better use of old and more recent antibacterial agents. Mortality is improved compared with historical series; however, long-term morbidity is significant particularly in the infant population. Further researches must be conducted to clarify specific aspects, such as anticonvulsant prophylaxis/therapy, and also for the improvement of microbiological diagnosis.

\section{Informed Consent}

Informed consent was obtained from all individual participants included in this study.

\section{Conflicts of Interest}

The authors declare no conflicts of interest regarding the publication of this paper.

\section{References}

[1] Brouwer, M.C., Tunkel, A.R., McKhann, G.M. II, et al. (2014) Brain Abscess. The New England Journal of Medicine, 371, 447-456. https://doi.org/10.1056/NEJMra1301635

[2] Marder, C.P. and Fink, K.R. (2014) Imaging of Intracranial Infections. In: Infections of the Central Nervous System, 4th Edition, Lippincott Williams \& Wilkins, Philadelphia, 24-47.

[3] Whitfield, P. (2005) The Management of Intracranial Abscesses. Advances in Clinical Neuroscience and Rehabilitation, 5, 12-15.

[4] Erdogan, E. and Cansever, T. (2008) Pyogenic Brain Abscess. Neurosurgical Focus, 24, E2. https://doi.org/10.3171/FOC/2008/24/6/E2

[5] Klein, M., Pfister, H.W., Tunkel, A.R., et al. (2014) Brain Abscess. In: Infections of the Central Nervous System, 4th Edition, Lippincott Williams \& Wilkins, Philadelphia, 522-549.

[6] Boom, W.H. and Tuazon, C.U. (1985) Successful Treatment of Multiple Brain Ab- 
scesses with Antibiotics Alone. Reviews of Infectious Diseases, 7, 189-199. https://doi.org/10.1093/clinids/7.2.189

[7] Sennaroglu, L. and Sozeri, B. (2000) Otogenic Brain Abscess: Review of 41 Cases. Otolaryngology_Head and Neck Surgery, 123, 751-755.

https://doi.org/10.1067/mhn.2000.107887

[8] Corson, M.A., Postlethwaite, K.P. and Seymour, R.A. (2001) Are Dental Infections a Cause of Brain Abscess? Case Report and Review of the Literature. Oral Diseases, 7, 61-65. https://doi.org/10.1034/j.1601-0825.2001.70112.x

[9] Vargas, J., Hernandez, M., Silvestri, C., et al. (2006) Brain Abscess Due to Arcanobacterium haemolyticum after Dental Extraction. Clinical Infectious Diseases, 42, 1810-1811. https://doi.org/10.1086/504436

[10] Limeres-Posse, J., Tomas-Carmona, I., Fernandez-Feijoo, J., et al. (2003) Abscesos cerebrales de origen oral. Revista de Neurología, 37, 201-206. https://doi.org/10.33588/rn.3703.2003192

[11] Ratnaike, T.E., Das, S., Gregson, B.A., et al. (2011) A Review of Brain Abscess Surgical Treatment-78 Years: Aspiration versus Excision. World Neurosurgery, 7, 431-436. https://doi.org/10.1016/j.wneu.2011.03.048

[12] Dake, M.D., McMurdo, S.K., Rosenblum, M.L., et al. (1986) Pyogenic Abscess of the Medulla Oblongata. Neurosurgery, 18, 370-372. https://doi.org/10.1227/00006123-198603000-00024

[13] Britt, R.H. and Enzmann, D.R. (1983) Clinical Stages of Human Brain Abscess on Serial CT Scans after Contrast Infusion. Computerized Tomographic, Neuropathological and Clinical Correlations. Journal of Neurosurgery, 59, 972-981. https://doi.org/10.3171/jns.1983.59.6.0972

[14] Sheehan, J.P., Jane, J.A., Ray, D.K.R., et al. (2008) Brain Abscess in Children. Neurosurgical Focus, 24, E6. https://doi.org/10.3171/FOC/2008/24/6/E6

[15] Chun, C.H., Johnson, J.D., Hofstetter, M., et al. (1986) Brain Abscess: A Study of 45 Consecutive Cases. Medicine, 65, 415-431. https://doi.org/10.1097/00005792-198611000-00006

[16] Chang, W.N. and Lui, C.C. (2006) Strategies for the Management of Bacterial Brain Abscess. Journal of Clinical Neuroscience, 13, 979-985.

https://doi.org/10.1016/j.jocn.2006.01.048

[17] Lee, T.H., Chang, W.N., Thung-Ming, S., et al. (2007) Clinical Features and Predictive Factors of Intraventricular Rupture in Patients Who Have Bacterial Brain Abscess. Journal of Neurology, Neurosurgery, and Psychiatry, 78, 303-309. https://doi.org/10.1136/jnnp.2006.097808

[18] Mampalam, T.J. and Rosenblum, M.L. (1988) Trends in the Management of Bacterial Brain Abscesses: A Review of 102 Cases over 17 Years. Neurosurgery, 23, 451-458. https://doi.org/10.1227/00006123-198810000-00008

[19] Fulgham, J.R., Wijdicks, E.F.M. and Wright, A.J. (1996) Cure of a Solitary Brainstem Abscess with Antibiotic Therapy: Case Report. Neurology, 46, 1451-1454. https://doi.org/10.1212/WNL.46.5.1451

[20] Frazier, J.L., Ahn, E.S. and Jallo, G.I. (2008) Management of Brain Abscesses in Children. Neurosurgical Focus, 24, E8. https://doi.org/10.3171/FOC/2008/24/6/E8

[21] Muzumdar, D., Jhawar, S. and Goel, A. (2011) Brain Abscess: An Overview. International Journal of Surgery, 9, 136-144. https://doi.org/10.1016/j.ijsu.2010.11.005 